\title{
Beitrag zur Kenntnis der Bildung echter Peroxyde des Eisens.
}

\author{
Von \\ G. Pelilini und D. Meneghint.
}

Es ist lange bekannt, dafs das Eisen sowohl im Ionenzustand wie auch in Form der undissozilierten Molekeln seiner Verbindungen eine wichtige katalytische Wirkung auf Oxydationsprozesse ausübt. Auf dieser Tatsache beruht eine der Reaktionen des Wasserstoffsuperoxyds, die sich mit Jodwasserstoffsäure oder Alkalijodiden in Gegenwart einer verdünnten Säure vollzieht. Es erfolgt eine langsame Reaktion mit freiwerdendem Jod, aber diese Reaktion findet mit viel gröfserer Geschwindigkeit statt, wenn man eine kleine Menge eines Eisensalzes hinzufügt. Eine solche Wirkung übt das Eisen auch in physiologischen Vorgängen aus, sowohl in den roten Blutkörperchen als auch in gewissen Pflanzenzellen, wo es mit Sicherheit nachgewiesen worden ist. Diese Wirkung steht mit der allgemeinen Eigenschaft der in lebenden Zellen enthaltenen ungesättigten Substanzen in Zusammenhang, welche die Fähigkeit aufweisen, den Luftsauerstoff aufzunehmen, und mit dem gemeinsamen Namen „Oxydasen" bezeichnet werden.

Den Mechanismus solcher Reaktionen erklärt man heute durch die Autoxydationstheorie, welche annimmt, dafs beim Oxydationsprozefs der autoxydierende Stoff einen Zwischenzustand eingeht, dem die Bildung eines Peroxyds entspricht.

Deshalb ist es einleuchtend, welche Wichtigkeit das Studium der Autoxydationserscheinungen des Eisens besitzt, ebenso wie die in den letzten Jahren gemachten Versuche die Bildung der intermediären Peroxyde direkt oder indirekt nachweisen.

Die ersten positiven Untersuchungen auf diesem Gebiete hat BRODE ${ }^{1}$ ausgeführt. Beim Studium der katalytischen Wirkung der

${ }^{1}$ Zeitschr. phys. Chem. 37 (1901), 303. 
Ferrosalze in der Reaktion zwischen Wasserstoff-superoxyd und Jodwasserstoffsäure vermutet er die Bildung eines sehr unbeständigen Eisenperoxyds. Diesen Schlufs leitet er aus der Beobachtung ab, dafs die Gefrierpunktserniedrigungen in den Ferrichlorid- und Wasserstoffsuperoxyd-Mischungen etwas gröfser als die berechnete sind; ferner dafs die von den Ferrosalzen durch Wasserstoffsuperoxyd hervorgerufene Färbung, oder des durch Ammoniak erhaltenen Niederschlags aus einer Ferrichloridlösung der Wasserstoffsuperoxyd hinzugefügt wurde, viel lebhafter ist, als jene, welche dem gewöhnlichen Ferrihydroxyd zukommt; endlich, dafs der Niederschlag eine grofse Menge Sauerstoff entwickelt. Trotzdem ist es Brope nicht gelungen, eine Verbindung peroxydischer Natur zu isolieren.

Etwas sichere Ergebnisse lieferte ein wenig später МАNCHOT, der ohne ein Eisenperoxyd isolieren zu können, dessen Existenz und das Atomverhältnis zwischen Eisen und Sauerstoff feststellte.

In seiner ersten Mitteilung ${ }^{1}$ über die Aktivierung des Sauerstoffs durch Ferrohydroxyd stellte er die Unmöglichkeit fest, bei der direkten Oxydation des Ferrohydroxyds an der Luft die primäre Bildung von Wasserstoffsuperoxyd oder seines Derivates wegen des raschen Verlaufes der sekundären Vorgänge, der Bildung des Ferrisalzes, nachzuweisen. Hingegen läfst sich die Bildung eines Peroxyds indirekt nachweisen, indem man dem System „FerrosalzAlkali-Sauerstoff" einen Acceptor (Alkaliarsenit) hinzufügt, welcher fähig ist, den aktiven Sauerstoff schneller als das Ferrohydroxyd anzunehmen. Das Verhältnis zwischen der aktivierten Sauerstoffmenge und der zur Bildung des Ferrihydrats entsprechende ist 1:1; daher ist die Bildung eines Peroxyds $\mathrm{FeO}_{2}$ sehr wahrscheinlich. Nach Englers Theorie ${ }^{2}$ wirkt das Eisenatom als Pseudoautoxydator, und der Vorgang der Reaktion ist der folgende:

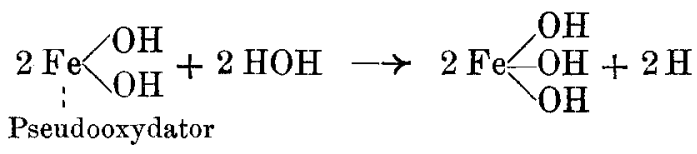

$$
2 \mathrm{H}+\mathrm{O}_{2} \rightarrow \mathrm{H}_{2} \mathrm{O}_{2}
$$

Bei der Autoxydation des Ferrohydroxyds soll sich also ein sekundäres Peroxydrat derselben Art bilden, wie jenes, deren Bildung

¿ Z. anorg. Chem. 27 (1901), 420.

2 Engler und Weissbera, Kritische Studien und die Vorgänge der Autoxydation, 1904, S. 106. 
durch Einwirkung von Wasserstoffsuperoxyd auf die Eisensalze angenommen werden kann. Diese Wirkung des Wasserstoffsuperoxyds nachzuweisen, ist der Zweck einer weiteren Mitteilung von MANCHot, ${ }^{1}$ deren Schlufsfolgerungen sich folgenderweise zusammenfassen lassen: Bei der Einwirkung des Wasserstoffsuperoxyds auf die Ferrosalze in neutraler Lösung in Gegenwart von Kaliumjodid und bei Temperatur von $0^{0}$ verbraucht ein Molekül Ferrosalz ungefähr anderthalb Molekül Wasserstoffperoxyd unter Bildung eines primären Peroxyds $\mathrm{Fe}_{2} \mathrm{O}_{5}$, welches für ein Atom Eisen je ein Atom aktiven Sauerstoff enthält. Die Bildung des Ferrisalzes ist keine direkte, vielmehr eine Folge dieses Autoxydationsprozesses. Auch die Ferrisalze sind fähig, den Sauerstoff (bzw. Wasserstoffsuperoxyd) anzunehmen, nur ist ihre Wirkungsweise viel langsamer als jene der Ferrosalze. Die freien Säuren verlangsamen die Oxydation der Ferrosalze, wie auch die Aufnahme des Sauerstoffs und des Wasserstoffsuperoxyds von seiten der Ferrisalze. Auf Grund dieser Untersuchung schreibt EngLer ${ }^{2}$ dem Peroxyd die Formel zu:

$$
\mathrm{OH}-\mathrm{O}-\mathrm{Fe}=\mathrm{SO}_{4} \rightarrow \mathrm{HO}-\mathrm{O}-\mathrm{Fe}<\underset{\mathrm{OH}}{\mathrm{OH}}
$$

weswegen:

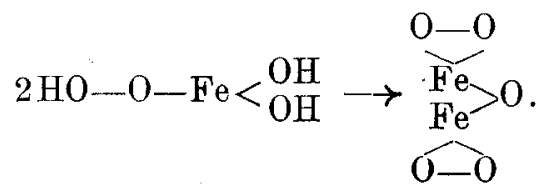

Luther und Schinow ${ }^{3}$ nehmen ebenfalls in ihrem Studium über Oxydations- und Reduktionskoppelungsprozesse an, dafs die Oxydation der Ferrosalze durch Wasserstoffsuperoxyd jener Koppelungsart angehöre, in welcher die Bildung einer Zwischenverbindung des Induktors angenommen wird. Dies findet bei dem Ferrosalze (Induktor) unter der Wirkung des Wasserstoffsuperoxyds oder des freien Sauerstoffs (Aktor) und bei Anwesenheit einer geeigneten Substanz, KJ,

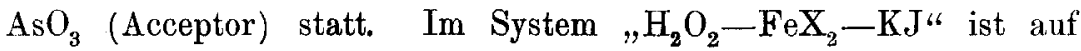
Grund von Manchoтs Ergebnisse folgendes Schema anzunehmen:

$$
\begin{aligned}
& \text { Aktor }+\mathrm{Fe}^{\mathrm{II}}=\mathrm{Fe}^{\mathrm{\gamma}}+\mathrm{Aktor} \text { (reductum) } \\
& \mathrm{Fe}^{\mathrm{V}}+\text { Acceptor }=\mathrm{Fe}^{\mathrm{III}}+\text { Acceptor (oxydatum). }
\end{aligned}
$$

2. c.

Z. auorg. Chem. Bd. 62. 
Eine sehr interessante Untersuchung über Autoxydation der Ferrosalze ist kürzlich von $\mathrm{JUST}^{1}$ geliefert worden. Er gelangt zu Resultaten, welche mit den Hypothesen Mavcrors und den darauffolgenden Beobachtungen ENGLERs in Widerspruch stehen. Er hat die Autoxydation des in Wasser gelösten Ferrobicarbonats rom Standpunkt der chemischen Kinetik untersucht, indem er die Konzentration der am Vorgang teilnehmenden Substanzen bestimmt. Die Kinetik lehrt, dals ein ganzes Sauerstoffmolekel an dem ersten Stadium der Oxydation teilnimmt. Die Kinetik vermag nicht zu bestimmen, in welcher Art der molekuläre Sauerstoff mit dem Ferrosalz reagiert, zeigt aber, dafs in dieser Reaktion ein und nicht zwei Moleküle des Ferrosalzes mit molekularem Sauerstoff in Reaktion treten. Infolge der hydrolytischen Abspaltung des Ferrobicarbonats

$$
\mathrm{Fe}\left(\mathrm{HCO}_{3}\right)_{2}=\mathrm{Fe}(\mathrm{OH})_{2}+2 \mathrm{CO}_{2}
$$

nimmt der hydrolytische Komponent $\mathrm{Fe}(\mathrm{OH})_{2}$ ein Sauerstoffmolekül auf, wobei er ein Zwischenprodukt bildet, nach EvGLER ein Moloxyd, nach Bredig ein Peroxydat der Formel:

$$
\stackrel{\mathrm{O}}{\mathrm{O}} \mathrm{\textrm {Fe }}(\mathrm{OH})_{2} \text {. }
$$

Dieses Peroxyd, im Gegensatz zu Manchots Annahme, ist ein vierwertiges Eisenderivat: es hat die gleiche Zusammensetzung wie Eisensäure, ist aber nicht identisch, weil die Eisensäure ein Derivat sechswertigen Eisens ist<smiles></smiles>

Die kinetische Methode gestattet also nicht die Hypothese Малснотs über die Zwischenbildung der Oxyde $\mathrm{FeO}_{2}, \mathrm{Fe}_{2} \mathrm{O}_{5}$, wohl aber jene eines Peroxyds $\mathrm{FeO}_{3}$ anzunehmen.

Die Darlegung der bis jetzt ausgeführten Studien über die wahrscheinlichen Eisenperoxyde hat uns zur vorliegenden Arbeit veranlafst, welche durch die Möglichkeit ein höheres Eisenoxyd vom Typus Wasserstoffsuperoxyd zu isolieren, die vorangegangenen Untersuchungen und im allgemeinen die Theorie der Autoxydation bestätigt.

${ }^{1}$ Just, Zeitschr. phys. Chem. 63, 385. 


\section{Experimenteller Teil.}

Die hier zu besprechenden experimentellen Ergebnisse beziehen sich auf die Wirkung des Wasserstoffsuperoxyds auf die Ferro- und Ferrisalze und die entsprechenden Hydroxyde. Da aus den vorausgehenden Versuchen von BRODE und von MaNchot und aus der allgemeinen Natur der peroxydischen Verbindungen, die sich bei Autoxydationsprozessen bilden, man höchst wahrscheinlich äufserst unbeständige Produkte zu erwarten hatte, folgten wir derselben Darstellungsmethode, welche uns zur Darstellung des Quecksilberperoxyds und des Nickelperoxyds geführt hatte. ${ }^{1}$

Die alkoholischen Lösungen von Ferro- oder Ferrichlorid und Suspensionen von Ferro- oder Ferrihydroxyd in absolutem Alkohol wurden auf -50 bis $-70^{\circ}$ abgekühlt und dann mit $30 \%$ igem Wasserstoffsuperoxyd reagieren gelassen, welche Temperatur die katalytischen Erscheinungen beinahe ausschlofs; bei den Lösungen von Chloriden wurde ferner eine alkoholische Lösung von Kalihydroxyd in genau solcher Menge zugesetzt, dafs sie zum Niederschlagen der gesuchten Superoxydverbindung ausreichte.

Wegen der Verdünnung mit Alkohol war die Konzentration des Wasserstoffsuperoxyds auf ungefähr $15 \%$ gesunken. Der Niederschlag wurde auf der Pumpe abgesaugt, zuerst mit stark abgekühlten Alkohol, dann mit Äther gewaschen.

Infolge der leichten Zersetzbarkeit der erhaltenen Niederschläge konnten wir dieselben keiner vollständigen quantitativen Analyse unterwerfen; deswegen haben wir uns darauf beschränkt, das Verhältnis $\mathrm{Fe}: \mathrm{O}$ zu bestimmen, das uns erlaubt, den Oxydationsgrad der Verbindung festzustellen. Daher wurde der Niederschlag sofort nach Isolierung und Waschung im geschlossenen Gefälse mit einer abgekühlten und durch Schwefelsäure leicht angesäuerten $1 / 10^{-}$-norm. Lösung von Kaliumjodid in Berührung gebracht und häufig geschüttelt, dann ungefähr 2 Stunden im Dunkeln gehalten. Nachdem die Lösung auf ein bestimmtes Volumen gebracht, wurde in einem Teil das Jod durch Erwärmen in Gegenwart von Salpetersäure auf Wasserbad verjagt, und dann das Eisen gravimetrisch durch Wägung als $\mathrm{Fe}_{2} \mathrm{O}_{3}$ bestimmt; in einem zweiten Teil bestimmten wir den aktiven Sauerstoff.

1 G. Peluint, Gaz\%. chim. ital. 38 I. (1908), 77. - G. Peldini u. D. Meneghini, Z. anorg. Chem. 60 (1908), 178. 
Letztere Bestimmung gründet sich auf die bekannte Reduktion, welche die Jodwasserstoffsäure auf die Eisensalze höherer Wertigkeit, als auf die der Form $\mathrm{FeX}_{2}$ angehörenden, hervorruft. Das freigewordene Jod titriert man mit einer $1 / 10^{\text {-norm. }} \mathrm{Na}_{2} \mathrm{~S}_{2} \mathrm{O}_{3}$-Lösung.

\section{A. Einwirkang des Wasserstoffsuperoxyds auf das Ferrohydroxyd.}

Eine Lösung von MoHrschem Salze in luftfreiem Wasser wurde jedesmal mit einer nicht ganz genügenden Menge von Kalilauge behandelt. Der hellgrüne Niederschlag des Hydroxyds wurde im Wasserstoffstrom filtriert und dann mit gekochtem Wasser gewaschen; darauf in einem grolsen Probierrohr in konzentriertem Alkobol suspendiert, dann in einem Dewarschen Gefäls mit festem Kohlensäureanhydrid und Aceton stark abgekühlt.

Sobald die Temperatur des Inhalts des Probierrohrs ca. $-60^{\circ}$ erreicht hatte, fügte man eine gewisse Menge abgekühltes $30 \%$ iges Wasserstoffsuperoxyd hinzu. Das Verhältnis der Reagenzien wurde nicht genau bestimmt: es war nur nötig, dals das Wasserstoffsuperoxyd in grofsem Überschufs sei gegenüber der für die Bildung eines Peroxyds $\mathrm{FeO}_{2}$, oder $\mathrm{Fe}_{2} \mathrm{O}_{5}$, oder $\mathrm{FeO}_{3}$ stöchiometrisch nötigen Menge.

$\mathrm{Zu}$ ungefähr $0.5 \mathrm{~g}$ Ferrohydroxyd gebrauchte man $5-10 \mathrm{ccm}$ $30 \%$ iges Wasserstoffsuperoxyd, dessen Konzentration jedoch wegen der Verdünnung mit Alkohol auf $10-15 \%$ sank.

Die Reaktionstemperatur schwankte zwischen $-20^{\circ}$ bis $-60^{\circ}$, wobei man immer bemüht war bei einer Temperatur zu arbeiten, welche ein Maximum der Bildungsgeschwindigkeit zugleich mit einem Minimum der katalytischen Wirkung gewährleistete.

Die Berührungsdauer des Hydroxyds mit dem Wasserstoffsuperoxyd wurde von Fall zu Fall geändert, um die Einwirkung der Zeitdauer auf die Reaktion zu beobachten.

Sobald das Ferrohydroxyd mit dem Wasserstoffsuperoxyd in Berührung kommt, ändert es seine hellgrüne Farbe in eine lebhaft hellrote, die durch die Zeit keine Änderung mehr erleidet.

Der Niederschlag wurde am Filter ausgeschlämmt und mit sehr kaltem Alkohol und dann mit Äther schnell gewaschen; mit Äther wurden gewöhnlich nur zwei Waschungen vorgenommen, weil er das Produkt viel mehr als der Alkohol zersetzt.

Versuch Nr. 1.

Dauer des Kontakts 15 Minuten. Ziemliche Zersetzung während der Waschung. $1 / 10^{-n o r m} . \mathrm{Na}_{2} \mathrm{~S}_{2} \mathrm{O}_{3}$ verbraucht $13.78 \mathrm{~cm}$ von freigewordenem Jod. 


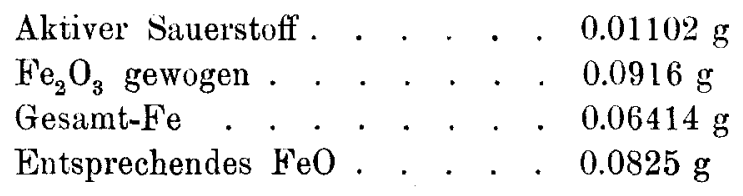

$\mathrm{Fe}: \mathrm{O}=1: 1.60$.

Versuch Nr. 2.

Dauer des Kontakts $1 \frac{1}{4}$ Stunde. - Zersetzung sehr gering. $1 / 10^{- \text {norm. }} \mathrm{Na}_{2} \mathrm{~S}_{2} \mathrm{O}_{3}$ verbraucht $10.6 \mathrm{ccm}$.

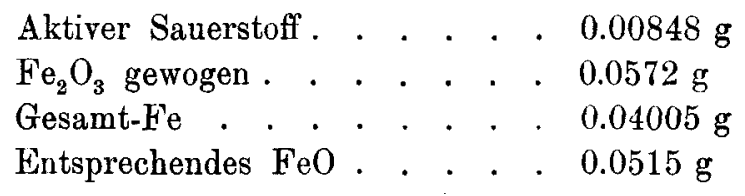

$\mathrm{Fe}: 0=1: 1.74$.

Versuch Nr. 3.

Dauer des Kontakts $1 \frac{3}{4}$ Stunde. - Zuletzt wurde mehrmals eine gewisse Menge Wasserstoffsuperoxyd zugesetzt. - Ziemliche Zersetzung während der Waschung. 1/10-norm. $\mathrm{Na}_{2} \mathrm{~S}_{2} \mathrm{O}_{3}$ verbraucht $43.46 \mathrm{ccm}$.

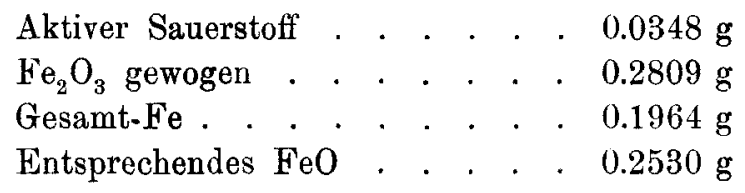

$$
\mathrm{Fe}: \mathrm{O}=1: 1.62 \text {. }
$$

Die so erhaltene Substanz ist ein echtes Peroxyd rom Typus des Wasserstoffsuperoxyds; es liefert nämlich alle dafür charakteristischen Reaktionen: mit Wasser und verdünnten Säuren zersetzt es sich unter Entwickelung von Sauerstoff und Bildung von Ferrihydroxyd bzw. Ferrisalz. In saurer Lösung reagiert es mit Titansulfat, mit Chromsäure, mit saurer Lösung von Kaliumpermanganat, mit Kaliumferricyanid und Ferrichlorid, in alkalischer Lösung mit Goldchlorid.

Die Reaktion von BaRReswrul gibt nicht immer ein sicheres Resultat; doch auch wenn der Äther sich nicht entschieden blau färbt, verändert die untenstehende Lösung ihre Farbe von gelb in grün. Übrigens ist bekannt wie empfindlich diese Reaktion und wie sehr der Gehalt der Reagenzien darauf Einflufs hat, und in 
unserem Falle die Spuren von in den folgenden Ätherwaschungen nicht ganz entferntem Alkohol.

Die übrigen Reaktionen und besonders die des Titansulfats und der Übermangansäure liefsen nie den leisesten Zweifel bestehen.

\section{B. Wirkung des Wasserstoffsuperoxyds auf alkoholische Lösungen von Ferrochlorid.}

Reines Ferrochlorid wurde in absolutem Alkohol gelöst, und während der Lösung ein Wasserstoffstrom durchgeleitet. Die so erhaltene Lösung von Ferrochlorid bleibt vollständig klar, wenn man sie von der Luft abschlielst; durch Sauerstoffaufnahme würde sie sich schnell durch Bildung von basischen Ferrisalzen trüben.

Eine solche Lösung (ca. $5 \mathrm{~g}$ von $\mathrm{FeCl}_{2}$ in $100 \mathrm{ccm}$ Alkohol) wurde stark abgekühlt $\left(-60^{\circ}\right)$, und ein Überschufs von $30 \%$ igem sehr kaltem Wasserstoffsuperoxyd zugesetzt. Der Zusatz mufs vorsichtig ausgeführt werden. Die gelbgrünliche Färbung des Ferrosalzes ändert sich sofort in intensiv rotbraun. Die Lösung bleibt vollständig klar und man beobachtet auch bei niedrigster Temperatur eine geringe Gasentwickelung.

Augenscheinlich erfolgt eine energische Reaktion, die Temperatur steigt plötzlich, und wenn der Zusatz von Wasserstoffsuperoxyd schnell geschieht, kann man nicht mehr die heftige Zersetzung aufhalten; in diesem Falle steigt die Temperatur ungeheuer. Setzt man nun dieser Lösung die genau nötige Menge einer alkoholischen Kalilösung hinzu, so wird eine dunkelrote Substanz niedergeschlagen, die auf die übliche Weise gewaschen, nach Austrocknung des Äthers als lebhaft hellrotes Pulver crscheint.

Auch in diesem Falle bemerkt man, dafs sofort nach Giefsen des Niederschlages auf den Filter, trotz der tiefen Temperatur eine katalytische Zersetzung eintritt und ein Geruch von Aldehyd sich bemerkbar macht. Nach den ersten Auslaugungen nimmt die Katalyse ein Ende. Bei Überschufs von Kalihydroxyd ist die Zersetzung eine starke, während man bei nicht hinreichendem Alkali beinahe keinen Niederschlag erhält. Die Lösung behält ihre intensive rotbraune Farbe lange Zeit auch bei gewöhnlicher Temperatur, geht nur schwer durch den Filter ohne Zersetzung, und erst nach einer gewissen Zeit bemerkt man im Filtrat einen Niederschlag von Ferrihydrat. Eine gleiche Erscheinung wurde auch von MANCHOT beobachtet. 


\section{Versuch Nr. 4.}

Dauer des Kontaktes nach Zusatz des Alkali 15 Minuten. Ziemliche Zersetzung während der Waschung. 1/10-110rm. $\mathrm{Na}_{2} \mathrm{~S}_{2} \mathrm{O}_{3}$ verbraucht $13.60 \mathrm{ccm}$.

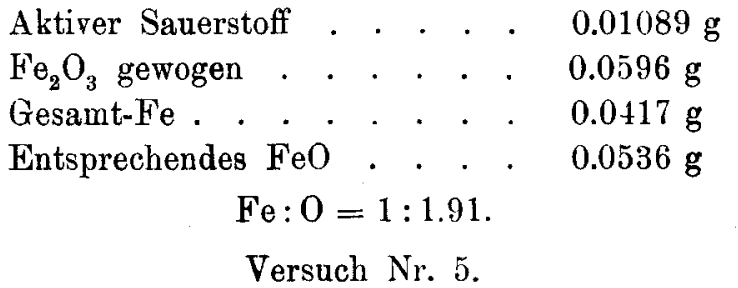

Dauer des Kontaktes 15 Minuten. Ziemliche Zersetzung. $1 / 10^{\text {-norm. }} \mathrm{Na}_{2} \mathrm{~S}_{2} \mathrm{O}_{3}$ verbraucht $20.98 \mathrm{ccm}$.

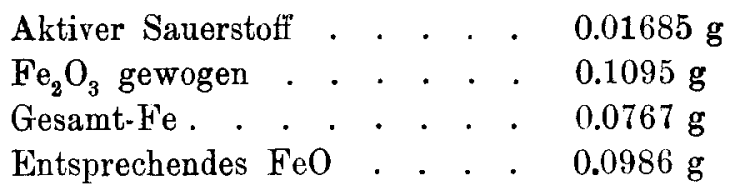

$\mathrm{Fe}: \mathrm{O}=\mathrm{I}: 1.77$.

Versuch Nr. 6.

Dauer des Kontaktes 1 Stunde. Zersetzung sehr gering. $1 / 10^{-n o r m} . \mathrm{Na}_{2} \mathrm{~S}_{2} \mathrm{O}_{3}$ verbraucht $12.5 \mathrm{ccin}$.

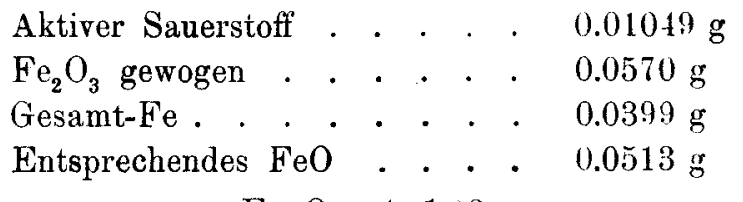

$$
\text { Fe: } \mathrm{O}=1: 1.92 \text {. }
$$

Versuch Nr. 7.

Dauer des Kontaktes 30 Minuten. Wegen eines geringen Mangels an Alkali ist der Niederschlag kolloidal. $1 / 10^{-n o r m} . \mathrm{Na}_{2} \mathrm{~S}_{2} \mathrm{O}_{3}$ verbraucht $3.2 \mathrm{ccm}$.

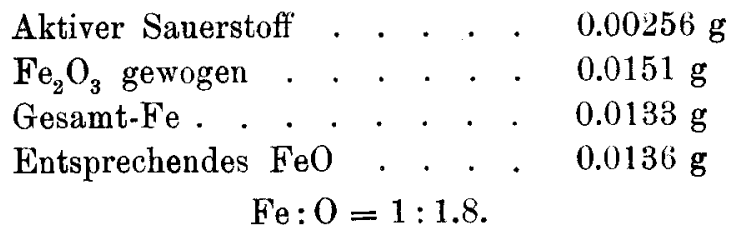

Den Niederschlag erhält man mit verschiedenen Mengen Kaliumchloroxyd vermischt, die jedoch nicht verhindern, dafs er alle Reaktionen der echten Peroxyde gibt. 


\section{Wirkung des Wasserstoffsuperoxyds auf Ferrihydrat.}

Eine lösung von reinem Ammoniumferrisulfat wurde mit Aznmoniak niedergeschlagen. Der gut gewaschene und noch feuchte Niederschlag wurde in Alkohol aufgeschwemmt und wie gewöhnlich mit Wasserstoff'superoxyd in Berührung gebracht. Nach 1 Stunde wurde der Niederschlag filtriert, aber die Filtrierung geschieht immer so langsam, dals sie niemals ein praktisches Resultat gegeben hat. Der Versuch wurde mehrmals mit negativem Resultat wiederholt, wegen des kolloidalen Zustandes des Niederschlages. Deswegen wurde das Hydroxyd kurze Zeit bei $150^{\circ}$ getrocknet; die schwärzliche Masse wurde schliefslich pulverisiert, und wie gewöhnlich mit Wasserstoffsuperoxyd zersetzt. So lange die alkoholische Suspension im Dewarschen Gefäls abgekühlt wurde, bemerkte man keine katalytische Erscheinung; diese beginnt am Filter. Das Filtrierte und mit Alkohol gewaschene Pulver erscheint glänzend schwarz.

Versuch Nr. 8.

Dauer des Kontaktes $2^{1 / 2}$ Stunde. 1/10-norm. $\mathrm{Na}_{2} \mathrm{~S}_{2} \mathrm{O}_{3}$ verbraucht $18.35 \mathrm{ccm}$.



$F_{\theta}: 0=1: 1.495$.

Versuch Nr. 9.

Dauer des Kontaktes 2 Stunden. ${ }^{1 / 10}$-norm. $\mathrm{Na}_{2} \mathrm{~S}_{2} \mathrm{O}_{3}$ verbraucht $65 \mathrm{cem}$.

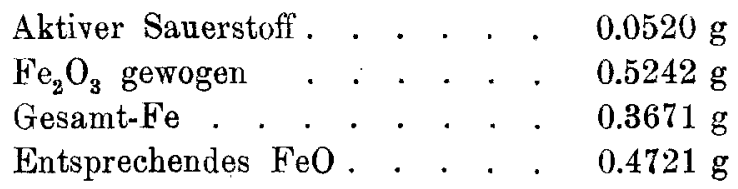

$\mathrm{Fe}: 0=1: 1.496$.

Aus diesen Versuchen zeigt sich, dals unter solchen Verhältuissen sich keine Peroxydverbindung bildet; die Reaktionen des Peroxyds sind tatsächlich alle negativ gewesen.

D. Wirkung des Wasserstoffsuperoxyds auf alkoholische Lösungen von Ferrichlorid.

Eine Lösung von reinem Ferrichlorid in absolutem Alkohol (ca. $8 \mathrm{~g}$ in $100 \mathrm{ccm}$ der Lösung) wurde stark abgekühlt, und nach- 
her die gewöhnliche Menge von Wasserstoffsuperoxyd hinzugefügt. Im Gegensatz zu Ferrolösungen zeigt sich in diesem Falle keine wahrnehmbare Veränderung der Farbe, und sowohl vor als nach dem Zusatz des Wasserstoffsuperoxyd geht die dunkel gelbrötliche Färbung der Lösung bei starker Abkühlung in bellstes Gelb über. Auch bei längerer Berührung ist weder eine Veränderung ersichtlich, noch irgend welche Vorsicht beim Zusatz des Wasserstoffsuperoxyds nötig; die Katalyse ist sehr schwach. Durch späteren Zusatz der zum völligen Niederschlag theoretisch nötigen Mengen von Kaliumhydrat in Alkohol erhält man ein ziemlich kolloidales dunkelrotes Präzipitat. Aus diesem Grunde filtriert es langsam, doch mit geringer Zersetzung. Wie gewöhnlich wurde die Waschung mit Alkohol und Äther ausgeführt.

\section{Versuch Nr. 10.}

Dauer des Kontaktes, nach Zusatz des Alkali 15 Minuten, Zersetzung sehr langsam. $1 / 10^{-n o r m} . \mathrm{Na}_{2} \mathrm{~S}_{2} \mathrm{O}_{3}$ verbraucht $7.14 \mathrm{ccm}$.

$$
\begin{aligned}
& \text { Aktiver Sauerstoff . . . . . } 0.00571 \mathrm{~g} \\
& \mathrm{Fe}_{2} \mathrm{O}_{3} \text { gewogen . . . . . } 0.0277 \mathrm{~g} \\
& \text { Gesamt-Fe . . . . . . . } 0.0194 \mathrm{~g} \\
& \text { Entsprechendes } \mathrm{FeO} \text {. . . . } 0.0255 \mathrm{~g}
\end{aligned}
$$

$$
\text { Fe }: 0=1: 1.93 \text {. }
$$

Versuch Nr. 11.

Dauer des Kontaktes 15 Minuten. 1/10-norm. $\mathrm{Na}_{2} \mathrm{~S}_{2} \mathrm{O}_{3}$ verbraucht $3.09 \mathrm{ccm}$.

$$
\begin{aligned}
& \text { Aktiver Sauerstoff . . . . . } 0.00247 \mathrm{~g} \\
& \mathrm{Fe}_{2} \mathrm{O}_{3} \text { gewogen . . . . . } 0.0127 \mathrm{~g} \\
& \text { Gesamt-Fe . . . . . . . } 0.0087 \mathrm{~g} \\
& \text { Entsprechendes } \mathrm{FeO} \text {. . . . } 0.0112 \mathrm{~g}
\end{aligned}
$$

Fe $: O=1: 1.9$.

Versuch Nr. 12.

Dauer des Kontaktes 30 Minuten. Zersetzung fast null. $1 / 10^{-n o r m . ~}$ $\mathrm{Na}_{2} \mathrm{~S}_{2} \mathrm{O}_{3}$ verbraucht $31.6 \mathrm{ccm}$.

$$
\begin{aligned}
& \text { Aktiver Sauerstoff . . . . . } 0.02528 \mathrm{~g} \\
& \mathrm{Fe}_{2} \mathrm{O}_{3} \text { gewogen . . . . . . } 0.1262 \mathrm{~g} \\
& \text { Gesamt-Fe . . . . . . } 0.0884 \mathrm{~g} \\
& \text { Entsprechendes } \mathrm{FeO} \text {. . . . } 0.1136 \mathrm{~g} \\
& \mathrm{Fe}: \mathrm{O}=1: 2 \text {. }
\end{aligned}
$$




\section{Versuch Nr. 13.}

Dauer des Kontaktes 1 Stunde. Ziemliche Zersetzung, während der Waschung geht in kolloidalen Zustand über. $1 / 10^{- \text {norm. }} \mathrm{Na}_{2} \mathrm{~S}_{2} \mathrm{O}_{3}$ verbraucht $14.17 \mathrm{ccm}$.

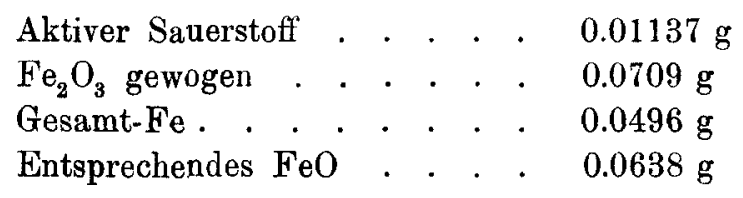

$\mathrm{Fe}: 0=1: 1.8$.

Versuch Nr. 14.

Dauer des Kontaktes 1 Stunde. Geringe Zersetzung. 1/10-norm. $\mathrm{Na}_{2} \mathrm{~S}_{2} \mathrm{O}_{3}$ verbraucht $15.2 \mathrm{ccm}$.

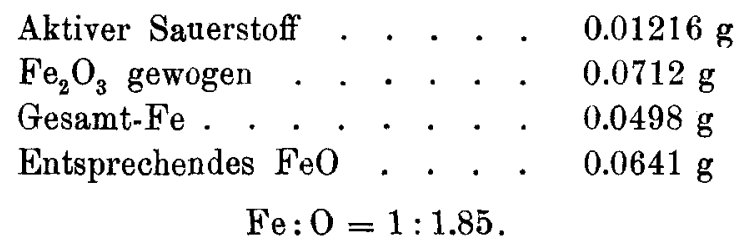

Der so erhaltene Niederschlag hat das gleiche Aussehen wie der von Ferrohydrat, und gibt alle Reaktionen von Wasserstoffsuperoxyd.

\section{E. Die Beständigkeit des Eisenperoxyds.}

Die Beständigkeit dieses Peroxyds ist sehr gering. Eine wie gewöhnlich vom Ferrochlorid erhaltene Probe, welche bein Waschen nur eine ganz geringe Zersetzung erlitt, gab, der Luft ausgesetzt, folgende Resultate:

\section{Reaktion der Titansäure:}

$$
\begin{aligned}
& \text { Frisch dargestellt. . . . . . positiv } \\
& \text { Nach } 5 \text { Minuten . . . . . positiv } \\
& \text {, } 1 \frac{1}{4} \text { Stunde. . . . . . unsicher } \\
& \text { " } 2 \text { Stunden . . . . . . negativ }
\end{aligned}
$$

Ein Teil derselben Probe hydrolysiert im Wasser. Nach 3 Stunden gibt das Wasser die Reaktion der Titansäure.

Eine mit Ferrochlorid erhaltene, und im trockenen Luftstrom ${ }^{1 /}{ }_{2}$ Stunde bei $0^{0}$ gehaltene Probe, hat bei der Analyse die folgenden Resultate gegeben: 
Versuch Nr. 15.

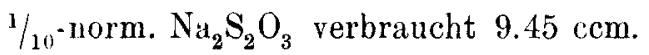

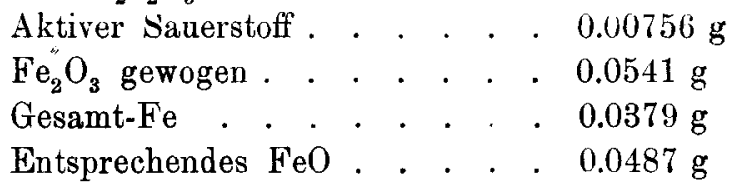

$\mathrm{Fe}: \mathrm{O}=1: 1.7$.

Eine mit Ferrichlorid erhaltene und im luftleeren Raume bei $0^{0}$ über Phosphorsäureanhydrid im Exsiccator gehaltene Probe hat nach 1 Stunde die folgenden Resultate gegeben:

Versuch Nr. 16.

$1 / 10$-norm. $\mathrm{Na}_{2} \mathrm{~S}_{2} \mathrm{O}_{3}$ verbraucht $11.8 \mathrm{ccm}$.



$\mathrm{Fe}: 0=1: 1.54$.

Als Endergebnis zeigt sich, dafs die Beständigkeit des Peroxyds sehr gering ist. Die Tatsache, dafs es noch nach 1 Stunde die charakteristischen Reaktionen der Peroxyde gibt, beweist immerhin klar, dafs diese sich auf Peroxyd beziehen und nicht durch unvollständige Auswaschung des Wasserstoffsuperoxyds hervorgerufen wird.

\section{F. Bestimmung des Wassergehaltes.}

Diese Bestimmung ist sehr interessant. Immerhin wäre es nötig eine Substanz zur Verfügung zu haben, deren Beständigkeit gröIser wäre als die von uns dargestellte. Wegen den Bedingungen der Bildung und des verschiedenen Wassergehaltes des mit Superoxyd gemischten Ferrihydroxyds, haben wir uns auf wenige Versuche beschränkt. Sie beziehen sich auf die in (15) und (16) angegebenen Versuche. Bei Versuch (15) entwickelten sich $0.0272 \mathrm{~g}$ Wasser bei einer Quantität $\mathrm{Fe}_{2} \mathrm{O}_{3}$ von $0.0621 \mathrm{~g}$ : bei $(16)=0.0254 \mathrm{~g}$ Wasser bei $0.0691 \mathrm{~g} \mathrm{Fe}_{2} \mathrm{O}_{3}$.

Das Produkt enthält also Wasser.

\section{Zusammenfassung der Resultate.}

Die folgende Tabelle umfalst die in dieser Mitteilung ențhaltenen analytischen Daten: 


\begin{tabular}{|c|c|c|c|c|c|c|c|}
\hline \multicolumn{2}{|c|}{ Versuche } & \multirow{2}{*}{$\begin{array}{c}\begin{array}{c}1 / 10-\text { norm. } \\
\mathrm{Na}_{2} \mathrm{~S}_{2} \mathrm{O}_{3} \\
\text { verbraucht } \\
\text { cem }\end{array} \\
13.78\end{array}$} & \multirow{2}{*}{$\begin{array}{c}\begin{array}{c}\text { Entsprech. } \\
\text { aktiver } \\
\text { Sauerstoff } \\
\mathrm{g}\end{array} \\
0.01102\end{array}$} & \multirow{2}{*}{$\begin{array}{c}\begin{array}{c}\mathrm{Fe}_{2} \mathrm{O}_{3} \\
\text { gewogen } \\
\mathrm{g}\end{array} \\
0.0916\end{array}$} & \multirow{2}{*}{$\begin{array}{c}\begin{array}{c}\text { Gesamt- } \\
\mathrm{Fe} \\
\mathrm{g}\end{array} \\
0.06414\end{array}$} & \multirow{2}{*}{$\begin{array}{c}\begin{array}{c}\text { Ent- } \\
\text { sprech. } \\
\text { FeO }\end{array} \\
0.0825\end{array}$} & \multirow{2}{*}{$\frac{\mathrm{Fe} / \mathrm{O}=}{\overline{1: 1.6}}$} \\
\hline 1 & $\mathrm{Fe}(\mathrm{OH})_{2}$ & & & & & & \\
\hline 2 & $n$ & 10.6 & 0.00848 & 0.0572 & 0.04005 & 0.0515 & $1: 1.74$ \\
\hline 3 & & 43.46 & 0.0348 & 0.2809 & 0.1964 & 0.2530 & $1: 1.62$ \\
\hline 4 & $\mathrm{FeCl}_{2}$ & 13.61 & 0.01089 & 0.0596 & 0.0417 & 0.0536 & $1: 1.91$ \\
\hline 5 & $"$ & 20.98 & 0.01685 & 0.1095 & 0.0767 & 0.0986 & $1: 1.77$ \\
\hline 6 & $"$ & 12.5 & 0.01049 & 0.0570 & 0.0399 & 0.0513 & $1: 1.92$ \\
\hline 7 &  & 3.2 & 0.00256 & 0.0151 & 0.0133 & 0.0136 & $1: 1.8$ \\
\hline 8 & $\mathrm{Fe}_{2} \mathrm{O}_{3}$ & 18.35 & 0.01475 & 0.1484 & 0.1040 & 0.1338 & $1: 1.495$ \\
\hline 9 & $"$ & 65.0 & 0.05200 & 0.5242 & 0.3671 & 0.4721 & $1: 1.496$ \\
\hline 10 & $\mathrm{FeCl}_{3}$ & 7.14 & 0.00571 & 0.0277 & 0.0194 & 0.0255 & $1: 1.93$ \\
\hline 11 & $"$ & 3.09 & 0.00247 & 0.0124 & 0.0087 & 0.0112 & $1: 1.9$ \\
\hline 12 & $"$ & 31.6 & 0.02528 & 0.1262 & 0.0884 & 0.1136 & $1: 2$ \\
\hline 13 & , & 14.17 & 0.01134 & 0.0709 & 0.0496 & 0.0638 & $1: 1.8$ \\
\hline 14 & $"$ & 15.2 & 0.01216 & 0.0712 & 0.0498 & 0.0641 & $1: 1.85$ \\
\hline 15 & $"$ & 9.45 & 0.00756 & 0.0541 & 0.0379 & 0.0487 & $1: 1.7$ \\
\hline 16 & $\mathrm{FeCl}_{2}$ & 11.8 & 0.00944 & 0.0872 & 0.0611 & 0.0786 & $1: 1.54$ \\
\hline
\end{tabular}

\section{Schlußfolgerung.}

Die in vorstehender Tabelle zusammengefalsten verschiedenen Versuche lassen uns folgern, dafs durch Einwirkung von Wassersitoffsuperoxyd auf Eisenverbindungen eine Substanz isoliert werden kann, welche die charakteristischen Merkmale der echten Peroxyde besitzt. Dieselbe ist von lebhaft hellroter Farbe, hat ein weniger kolloidales Aussehen als niedergeschlagenes Ferrihydrat und ist sehr un. beständig. Ihre Zusammensetzung kann nicht ganz sicher bestimmt werden: erstens wegen der Unbeständigkeit der Verbindung, zweitens wegen der Anwesenheit von Ferrihydrat in verschiedener Menge, endlich weil die durch Alkali aus Ferro- und Ferrichloriden dargestellten Niederschläge immer mit Alkalichlorid verunreinigt sind.

Auf Grund der von МАNCHOT und von JosT ausgeführten Studien kann das Verhältnis $\mathrm{Fe}: \mathrm{O}$ nicht mehr als 1:3 und nicht weniger als $1: 2$ betragen.

Die von uns erhaltenen Produkte konnten deshalb eine Mischung von den möglichen Peroxyden und $\mathrm{Fe}_{2} \mathrm{O}_{3}$ darstellen. Immerhin ist es bemerkenswert, dal's es nie gelungen ist das Verhältnis $1: 2 \mathrm{zu}$ überschreiten; selbst im günstigsten Falle, d. h. bei der Anwendung der Ferrisalze nicht. Im Versuch (12) ist das Verbältnis genau 
$1: 2$. Betrachten wir nun, dafs bei der Filtrierung des entsprechenden Niederschlages eine, wenn auch nur geringe Zersetzung stattfand, so könnte man zu der Annahme geführt sein, dafs das Verbältnis $1: 2$ hier überschritten ist. Angenommen das Verhältnis sei $1: 2$, so wäre die Zusammensetzung des Eisenperoxyds $\mathrm{Fe}_{2} \mathrm{O}_{2}$ oder $\mathrm{Fe}_{2} \mathrm{O}_{4}$ und die Konstitution:

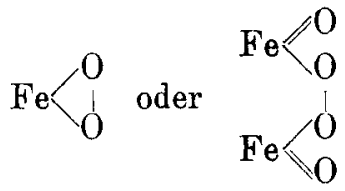

Es ist ferner noch das ganz verschiedene Verhalten des Ferrochlorids und Ferrichlorids mit Wasserstoffsuperoxyd bemerkenswert. Die Lösung von Ferrosalz in Alkohol ist von grüngelblicher Färbung und diese nimmt durch starke Abkühlung leicht an Intensität $a b$; die von Ferrisalz ist gelbbraun gefärbt und die Farbe wird durch Abkühlung ersichtlich abgeschwächt. Die Lösung von Ferrosalz reagiert kräftig mit Wasserstoffsuperoxyd unter starker Wärmeentwickelung und die intensive braune Färbung zeigt bei $\mathrm{Ab}$ kühlung keine nennenswerte Abschwächung. Die Lösung von Ferrisalz reagiert nicht (oder sehr schwach) mit Wasserstoffsuperoxyd. Die Farbe ist nur wenig von jener der Ferrisalze für sich allein verschieden und nimmt so wie dieses durch Abkühlung an Intensität ab. Dieses verschiedene Verhalten der zwei Salztypen beweist sicherlich, dafs zwischen dem Ferrosalze und Wasserstoffsuperoxyd eine chemische Verbindung eintritt, über deren Natur wir nichts Bestimmtes sagen können und dafs das Ferrisalz eine solche Verbindung nicht oder nur sehr langsam einzugehen vermag, auch unter Berücksichtigung der tiefen Temperatur, bei welcher die Arbeiten ausgeführt wurden.

Das in diesem Falle sich bildende Ferriperoxyd entsteht aus der Wirkung von Wasserstoffsuperoxyd auf Ferrihydroxyd in statu nascendi und nicht durch eine vorher bestehende Peroxydverbindung, wie es beim Ferrosalz der Fall zu sein scheint.

Padua, Chemisches Institut der Universität, März 1909.

Bei der Redaktion eingegangen am 22. März 1909. 